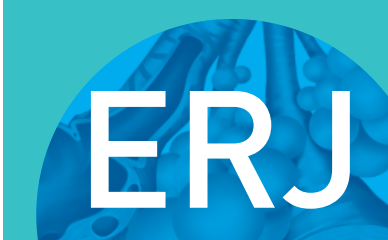

open research

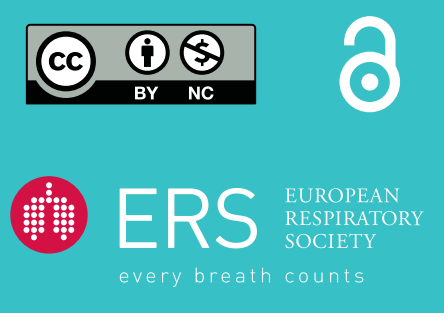

\section{Health-related quality of life and psychological wellbeing are poor in children with bronchiectasis and their parents}

\section{To the Editor:}

Bronchiectasis is characterised by chronic wet cough, a cycle of inflammation, impaired mucociliary clearance, bacterial colonisation and infection [1]. People with bronchiectasis experience substantial disease burden, including recurrent hospitalisations and impaired quality of life (QoL) [2]. The prevalence and burden of bronchiectasis is increasingly appreciated worldwide [3].

Families with children who have chronic illnesses have a greater likelihood of experiencing poor psychological health [2,4]. Examining the impact of a childhood illness on the psychological well-being of family members, particularly the primary caregiver, is important as maternal depression may influence nonadherence to therapy and overall morbidity $[5,6]$. Among children with bronchiectasis, health-related QoL (HRQoL), parental stress and coping are negatively affected during respiratory exacerbations [2]. However, there is little data on child and parent perception of HRQoL or the psychological wellbeing of parents of children with stable-state bronchiectasis compared with healthy children. A previous report found no difference in anxiety or depression scores but reported poorer physical health QoL in children with bronchiectasis compared with controls [7]. However, this assessment was not undertaken in conjunction with parental reports of psychological health. In this study we sought to determine: 1) self-reported and parent-proxy HRQoL in children with clinically stable bronchiectasis, compared with age-matched peers; and 2) parent or caregivers' psychological well-being.

Children (aged 5-18 years) with clinically stable bronchiectasis were recruited from outpatient clinics at two Australian tertiary hospitals. All parent participants provided written, informed consent, and assent (in children $>12$ years of age) was also obtained. Children and their parents completed an age-appropriate version of the Pediatric Quality of Life Inventory (PedsQL), a generic HRQoL scale [8]. Items on the PedsQL generate a total score and two summary scores: physical health (physical health subscore) and psychosocial health (emotional, social and school functioning subscores), with higher scores representing better HRQoL. Parents also completed: the Hospital Anxiety and Depression Scale (HADS) and the Center for Epidemiologic Studies Depression Scale (CES-D) [9, 10], with higher scores representing greater depression and anxiety; and the parent-proxy QoL questionnaire of paediatric chronic cough (PC-QoL), with lower scores reflecting greater cough frequency, concerns or worries [11].

Age-matched control children (with no diagnosis of a respiratory condition) were recruited from amongst the children and contacts of staff within the recruiting hospitals, as well as the community. They and their parents completed the same questionnaires (with the exception of the PC-QoL).

The study was approved by the relevant human research ethics committees (HREC/15/QRCH/69 and HREC/15/WCHN/125).

@ERSpublications

Children with bronchiectasis in a stable state have poor HRQoL and their parents report higher levels of anxiety and depression. Parental perception of HRQoL is associated with poorer parental psychological wellbeing. http://bit.ly/2lcf3uB

Cite this article as: Cox NS, Wilson CJ, Bennett KA, et al. Health-related quality of life and psychological wellbeing are poor in children with bronchiectasis and their parents. ERJ Open Res 2019; 5: 00063-2019 [https://doi.org/10.1183/23120541.00063-2019].

Copyright $\odot$ ERS 2019. This article is open access and distributed under the terms of the Creative Commons Attribution NonCommercial Licence 4.0. 
Statistical analyses were undertaken using SPSS software (v25.0; IBM Corp., Armonk, NY, USA). Descriptive statistics are presented as mean \pm SD or median (interquartile range). Categorical variables are reported descriptively using frequency (n) and proportion (\%). Differences between groups for continuous data were analysed using the Mann-Whitney $U$ test and the Chi-squared test for categorical data. Relationships between HRQoL and psychological well-being variables were explored using correlation coefficients (Pearson or Spearman's rank depending on normality distribution), with correlations interpreted as excellent (>0.75), moderate (0.5-0.75), fair (0.25-0.50) and no relationship (0.00-0.25) [12].

41 children with bronchiectasis (mean \pm SD age $10 \pm 4$ years, body mass index (BMI) $\mathrm{z}$-score $0.62 \pm 1.02$ ) and their 41 parents, and 41 control children and their 41 parents were included in this study. Control children were age-matched, with similar BMI z-scores (0.62 \pm 1.01$)$. Most children with bronchiectasis undertook airway clearance therapy daily (73\%) and engaged in daily exercise (67\%). Mean forced expiratory volume in $1 \mathrm{~s}\left(\mathrm{FEV}_{1}\right)$ was $91 \pm 16 \%$ predicted (range $\left.51-117 \%\right)$.

Children with bronchiectasis had significantly worse self-reported and parent-perceived HRQoL compared with their healthy peers (table 1). In the bronchiectasis group, fair-to-moderate correlations between parent-proxy and child PedsQL physical, psychosocial and total scores were seen $\left(\mathrm{r}_{\mathrm{s}}\right.$ range $0.36-0.66$; all $\mathrm{p}<0.01)$. There was a fair correlation between disease severity $\left(\mathrm{FEV}_{1} \%\right.$ pred) and Parent PedsQL physical score $\left(r_{s}=0.482\right)$. In the control group, the only statistically significant association was between the parent and child's PedsQL psychosocial summary score $\left(r_{s}=0.41, p=0.009\right)$. All others ranged $r_{s}=-0.036-0.005$.

Nearly three times as many parents of children with bronchiectasis reported symptoms of anxiety (49\%) compared with parents of healthy controls $(17 \%)(\mathrm{p}=0.009)$. In parents of children with bronchiectasis, worse HRQoL (PedsQL total score) was associated with poorer parental psychological health (HADS depression and CES-D, both $\left.\mathrm{r}_{\mathrm{s}}>-0.45, \mathrm{p}<0.01\right)$. Similarly, poorer PC-QoL was significantly associated with anxiety $\left(\mathrm{r}_{\mathrm{s}}=-0.40, \mathrm{p}=0.03\right)$ and depression on the CES-D $\left(\mathrm{r}_{\mathrm{s}}=-0.32, \mathrm{p}=0.01\right)$ but did not relate to depression on HADS $\left(\mathrm{r}_{\mathrm{s}}=-0.17, \mathrm{p}=0.33\right)$.

Our study is novel for several reasons. Firstly, we report on both parent-proxy and child QoL concurrently, while previous studies reported either child or parent-proxy reports and found poor HRQoL in stable-state [7] and during an exacerbation [2]. Our finding of a significant association between parent and child reports of HRQoL, suggests that, while not equivalent, in the absence of a validated disease-specific measure for children, self-report or parent-proxy measures of HRQoL may be used depending on child's age and capacity, and reasonably reflect disease impact. This may be less applicable to a healthy population; our few correlations between parental and healthy child QoL are consistent with previous findings [13].

Secondly, the higher prevalence of anxiety and depression in parents of children with stable-state bronchiectasis compared with controls, is consistent with reports of parents of children with cystic fibrosis (CF) [14]. Poorer parental psychological health may be related to the high treatment burden of their children, with nearly $75 \%$ of child participants with bronchiectasis in this study engaged in regular airway clearance therapy and exercise. High treatment burden is recognised in adults with bronchiectasis [15].

\begin{tabular}{|c|c|c|c|}
\hline & Bronchiectasis & Controls & p-value \\
\hline \multicolumn{4}{|l|}{ Children } \\
\hline Males:females $n$ & $23: 18$ & $20: 21$ & $0.557^{\#}$ \\
\hline PedsQL physical & $75(63-91)$ & 88 (84-94) & $0.002^{\pi}$ \\
\hline PedsQL psychosocial & $17(16-19)$ & $63(47-78)$ & $<0.001^{\Re}$ \\
\hline PedsQL total & $67.4(58.7-81.5)$ & 88 (85-95) & $<0.001^{\Uparrow}$ \\
\hline \multicolumn{4}{|l|}{ Parents } \\
\hline PedsQL physical & $75(54.7-90.6)$ & $94(88-100)$ & $<0.001^{\text {ๆ }}$ \\
\hline PedsQL psychosocial & 17 (15-18) & $62(51-73)$ & $<0.001^{\Uparrow}$ \\
\hline PedsQL total & $65.8(54.1-80.7)$ & $95(88-101)$ & $<0.001^{\pi}$ \\
\hline HADS anxiety & $20(49)$ & 7 (17) & $0.009^{\#}$ \\
\hline HADS depression & 13 (32) & $1(2)$ & $0.001^{\#}$ \\
\hline CES-D & $23(56)$ & $1(2)$ & $<0.001^{\#}$ \\
\hline
\end{tabular}


This finding is important given the known association between anxiety, depression and treatment adherence described in other respiratory populations $[5,6]$. While our small dataset did not allow an evaluation of this relationship, future exploration of the link between treatment burden, adherence and the psychological health of parents of children with bronchiectasis, and a comparison of this to children with $\mathrm{CF}$ is warranted.

The poorer parental psychological well-being reported by parents of children with bronchiectasis highlights the importance of a "whole-of-family" approach to screening and management for bronchiectasis, including current recommendations for psychosocial interventions for families of children with bronchiectasis [3]. Adolescents with CF are twice as likely to report depression and/or anxiety if a parent has psychological distress [14]. Whether parental psychological distress serves as a precursor to the development of child psychological distress is unclear.

The small participant numbers in this study may serve to under- or overestimate the degree of impairment in HRQoL experienced by children with bronchiectasis, as well as the prevalence of parental psychological distress. Children with bronchiectasis in this study had mild disease, with exacerbation frequency or antibiotic prescription unknown; in addition, only stable-state children with bronchiectasis were included. Therefore, the extent these findings apply to those with severe disease, or with an acute exacerbation, are unclear. In the absence of information regarding exacerbations and hospitalisations, their contribution to poorer HRQoL are unknown. Participants with bronchiectasis were recruited from two geographical and climatic regions, whilst control participants came from geographically disparate locations. Whether environmental and cultural differences influence QoL or psychological well-being cannot be accounted for.

In comparison with their healthy peers, children with bronchiectasis have poor HRQoL and their parents report higher levels of anxiety and depression. Attention to, and strategies to improve, HRQoL and parental psychological well-being are warranted in this patient group.

Narelle S. Cox ${ }^{1,2}$, Christine J. Wilson ${ }^{3}$, Katharine A. Bennett ${ }^{4}$, Kylie Johnston ${ }^{5}$, Angela Potter ${ }^{6}$, Anne B. Chang ${ }^{7,8,9}$ and Annemarie L. Lee $e^{1,2,10,11}$

${ }^{1}$ Discipline of Physiotherapy, La Trobe University, Melbourne, Australia. ${ }^{2}$ Institute for Breathing and Sleep, Melbourne, Australia. ${ }^{3}$ Physiotherapy, Queensland Children's Hospital, Brisbane, Australia. ${ }^{4}$ Physiotherapy, Royal Children's Hospital, Melbourne, Australia. ${ }^{5}$ School of Health Sciences, University of SA, Adelaide, Australia. ${ }^{6}$ Physiotherapy, Women's and Children's Hospital, Adelaide, Australia. ${ }^{7}$ Dept of Respiratory and Sleep Medicine, Queensland Children's Hospital, Brisbane, Australia. ${ }^{8}$ Child Health Division, Menzies School of Health Research, Charles Darwin University, Darwin, Australia. ${ }^{9}$ Centre for Children's Health Research, Queensland University of Technology, Brisbane, Australia. ${ }^{10}$ Centre for Allied Health Research and Education, Cabrini Health, Melbourne, Australia. ${ }^{11}$ Dept of Physiotherapy, Monash University, Melbourne, Australia.

Correspondence: Annemarie L. Lee, Dept of Physiotherapy, Monash University, McMahons Road, Frankston, Vic 3199, Australia. E-mail: Annemarie.Lee@monash.edu

Received: 07 March 2019 | Accepted after revision: 09 July 2019

Support statement: N.S. Cox is supported by a National Health and Medical Research Council (NHMRC) early career fellowship (GNT1119970). A.B. Chang is supported by a NHMRC practitioner fellowship (GNT1154302) and holds multiple NHMRC grants awarded related to diseases associated with paediatric cough and bronchiectasis.

Conflict of interest: None declared.

\section{References}

1 Goyal V, Grimwood K, Marchant J, et al. Pediatric bronchiectasis: no longer an orphan disease. Pediatr Pulmonol 2016; 51: 450-469.

2 Kapur N, Masters IB, Newcombe P, et al. The burden of disease in pediatric non-cystic fibrosis bronchiectasis. Chest 2012; 141: 1018-1024.

3 Chang AB, Bush A, Grimwood K. Bronchiectasis in children: diagnosis and treatment. Lancet 2018; 392: 866-879.

4 Quittner AL, Espelage DL, Opipari LC, et al. Role strain in couples with and without a child with a chronic illness: associations with marital satisfaction, intimacy, and daily mood. Health Psychol 1998; 17: 112-124.

5 Bartlett SJ, Krishnan JA, Riekert KA, et al. Maternal depressive symptoms and adherence to therapy in inner-city children with asthma. Pediatrics 2004; 113: 229-237.

6 Perry CD. Does treating maternal depression improve child health management? The case of pediatric asthma. J Health Econ 2008; 27: 157-173.

7 Bahali K, Gedik AH, Bilgic A, et al. The relationship between psychological symptoms, lung function and quality of life in children and adolescents with non-cystic fibrosis bronchiectasis. Gen Hosp Psychiatry 2014; 36: 528-532. 
8 Varni JW, Seid M, Kurtin PS. PedsQL 4.0: reliability and validity of the Pediatric Quality of Life Inventory version 4.0 generic core scales in healthy and patient populations. Med Care 2001; 39: 800-812.

9 Radloff LS. The use of the Center for Epidemiological Studies Depression Scale in adolescents and young adults. J Youth Adolesc 1991; 20: 149-166.

10 Zigmond AS, Snaith RP. The hospital anxiety and depression scale. Act Psychiatr Scand 1983; 67: 361-370.

11 Newcombe PA, Sheffield JK, Juniper EF, et al. Validation of a parent-proxy quality of life questionnaire for paediatric chronic cough (PC-QOL). Thorax 2010; 65: 819-823.

12 Portney L, Watkins M. Correlation. In: Portney L, Watkins M, eds. Foundations of Clinical Research. 3rd Edn. New Jersey, Pearson, Prentice Hill, 2009; pp. 523-538.

13 Upton P, Maddocks A, Eiser C, et al. Development of a measure of the health-related quality of life of children in public care. Child Care Health Dev 2005; 31: 409-415.

14 Quittner AL, Goldbeck L, Abbott J, et al. Prevalence of depression and anxiety in patients with cystic fibrosis and parent caregivers: results of The International Depression Epidiological Study across nine studies. Thorax 2014; 69: 1090-1097.

15 McCullough AR, Tunney MM, Quittner AL, et al. Treatment adherence and health outcomes in patients with bronchiectasis. BMC Pulm Med 2014; 14: 107. 